\title{
A Square Root Unscented Kalman Filter for visual monoSLAM
}

\author{
Steven Holmes, Georg Klein and David W Murray
}

\begin{abstract}
This paper introduces a Square Root Unscented Kalman Filter (SRUKF) solution to the problem of performing visual Simultaneous Localization and Mapping (SLAM) using a single camera. Several authors have proposed the conventional UKF for SLAM to improve the handling of non-linearities compared with the more widely used EKF, but at the expense increasing computational complexity from $O\left(N^{2}\right)$ to $O\left(N^{3}\right)$ in the map size, making it unattractive for video-rate application. Van der Merwe and Wan's general SRUKF delivers identical results to a general UKF along with computational savings, but remains $O\left(N^{3}\right)$ overall. This paper shows how the SRUKF for the SLAM problem can be re-posed with $O\left(N^{2}\right)$ complexity, matching that of the EKF. The paper also shows how the method of inverse depth feature initialization developed by Montiel et al. for the EKF can be reformulated to work with the SRUKF. Experimental results confirm that the SRUKF and the UKF produce identical estimates, and that the SRUKF is more consistent than the EKF. Although the complexity is the same, the SRUKF remains more expensive to compute.
\end{abstract}

\section{INTRODUCTION}

The workhorse of recursive visual structure from motion and visual simultaneous localization and mapping has for many years been the Extended Kalman Filter (EKF) (e.g. [1], [2], [3], [4]). Given the Bayesian pedigree of the Kalman filter for Gaussian pdfs, and the engineer's predilection for linearizing systems about operating points, it is a short and intuitive leap to the extended filter. However, as several authors have noted [5], [6], [7], it is also a dangerous leap. The EKF can be unacceptably biased and inaccurate, and lead to inconsistency; and they argue that the EKF's position as algorithm of first resort for estimating the state of nonlinear dynamical systems should not make it the algorithm of last resort. Nor does the cost of computing the EKF scale so favourably with state size that it can automatically be excused its weaknesses elsewhere.

The Unscented Transform was introduced by Julier, Uhlmann and Durrant-Whyte [8], [5] as a method of avoiding bias in non-linear transforms. A number of authors have applied the straight Unscented KF to SLAM problems [9], [10], [11]. Most pertinent here, Chekhlov et al. [12] applied it to visual SLAM using a single camera. However, their motivation appeared to be that of implementational convenience, and the authors reported neither the method's accuracy vis-àvis the EKF, nor its computational complexity. Although their particular experiment ran at video rate, the UKF's $O\left(N^{3}\right)$

The authors are with the Active Vision Laboratory, Department of Engineering Science, University of Oxford, Oxford OX1 3PJ, UK. www.robots.ox.ac.uk/ActiveVision [sah,gk,dwm]@ robots.ox.ac.uk

This work was supported by Grants GR/S97774 and EP/D037077 from the UK Engineering and Physical Science Research Council. SAH is supported by an EPRSC Research Studentship. The authors are grateful for conversations with Professor Gene H Golub and Dr Simon Julier. complexity in the map size rules out its use for other than very small maps. Andrade-Cetto et al. [10] partially finessed the UKF's complexity problem by exploiting the linearity of the map state update and applying the UKF only to the state members describing their vehicle position. However, they did not consider non-linearity in the measurement process.

In this paper we propose a Square Root Unscented Kalman Filter (SRUKF) for monocular visual SLAM. The general SRUKF was introduced by van der Merwe and Wan [6]. They showed that propagating the square root of the covariance avoided the UKF's Cholesky decompositions and hence made substantial computation savings. However, the overall complexity stuck at $O\left(N^{3}\right)$ because of a remaining QR decomposition. A key result here, developed in Section IV, is that by careful re-arrangement of the state and re-setting of the QR algorithm, the SRUKF can be be applied to SLAM with $O\left(N^{2}\right)$ complexity. Also shown is how to deal with the rank deficiency introduced when adding an inverse depth point to the map in the SRUKF.

Experiments show (i) that the SRUKF and UKF produce identical structure and motion of the scene and the camera; (ii) that the SRUKF produces more consistent estimates than the EKF; and (iii) that while the SRUKF has quadratic complexity it still has a computational cost of an order of magnitude greater than the EKF.

\section{Monocular Visual SLAM}

We are concerned with the estimation of the state $x$ and covariance $\mathrm{P}$ of a discrete-time non-linear dynamical system, whose evolution is modelled from time step $k$ to $k+1$ as

$$
\boldsymbol{x}_{k+1}=\boldsymbol{F}\left(\boldsymbol{x}_{k}, \boldsymbol{v}_{k}, \boldsymbol{u}_{k}\right),
$$

through observations related to the state through a vector of non-linear functions

$$
\boldsymbol{y}_{k}=\boldsymbol{H}\left(\boldsymbol{x}_{k}, \boldsymbol{n}_{k}\right) .
$$

Here, the known control input is $\boldsymbol{u}_{k}$, and and the process and measurement noises are $\boldsymbol{v}_{k}$ and $\boldsymbol{n}_{k}$.

For monocular visual SLAM, as described by Davison et al. [13], the state vector comprises two parts, the varyingsize set of 3D locations of map features $\left\{\boldsymbol{X}^{i}\right\}, i=1 \ldots m$, which are assumed fixed over time, and the time dependent camera pose and motion $\left\{\boldsymbol{O}_{k}, \boldsymbol{V}_{k}\right\}$. A constant velocity model is assumed where, at each time step, white Gaussian distributed impulses in velocity and angular velocity are applied. The average velocities during a time step, $\boldsymbol{V}_{k}+$ $\boldsymbol{v}_{k} / 2$, are integrated over each timestep to give the changes in position and orientation. The measurements are assumed 
to arise from a perspective projection of the scene points into the camera

$$
\left[\begin{array}{c}
\boldsymbol{y}_{k}^{i} \\
1
\end{array}\right] \sim \mathrm{C}_{k}[\mathrm{I} \mid \mathbf{0}] \mathrm{E}\left(\boldsymbol{O}_{k}\right)\left[\begin{array}{c}
\boldsymbol{X}_{k}^{i} \\
1
\end{array}\right]
$$

where $\mathrm{C}_{k}$ is the camera's intrinsic calibration which is changeable but assumed known. (For live experimentation a correction for radial distortion is applied of course.)

The matrix $\mathrm{E}\left(\boldsymbol{O}_{k}\right)$ representing the rigid body transformation from world to camera frame is updated after both the prior calculation and the posterior update. The covariances are also transformed into the current coordinate frame. In Kalman filter-based methods this is done by first transforming the probability density function through the nonlinear system model then updating the probability density using the observations. To do this, the probability density functions are assumed to be Gaussians. These transformations are approached in two ways for SLAM. The EKF linearises the functions about the current state whereas the UKF approximates the probability density function with a set of deterministically selected "sigma points" and passes them through the nonlinear function. The transformed sigma points then can be used to calculate the transformed probability density [5].

\section{The SQUARE Root UKF}

While it is claimed that the UKF gives better results than the EKF, the utility of the standard implementation is wholly compromised for video-rate applications by the Cholesky decomposition required to generate the sigma points at each time step, the complexity of which scales as $O\left(N^{3}\right)$. Recently, van der Merwe and Wan [6] introduced the Square Root UKF, a re-implementation of the general UKF which delivers exactly the same results (to within machine accuracy), but which cunningly avoids the decomposition by directly propagating the Cholesky factor $S$ rather than the covariance $\mathrm{P}$. While providing substantial savings, as mentioned above, the general SRUKF remains $O\left(N^{3}\right)$ for state estimation problems ${ }^{1}$. We need now to describe the SRUKF in order to understand the changes made to it later for $O\left(N^{2}\right)$ SLAM.

\section{A. Predicting the state}

At the start of the update cycle from $k$ to $k+1$, let $\mathrm{S}_{k \mid k}$ be the upper Cholesky factor in the decomposition $\mathrm{S}_{k \mid k}{ }^{\top} \mathrm{S}_{k \mid k} \leftarrow$ $\mathrm{P}_{k \mid k}$, and let $\mathrm{T}_{k}$ and $\mathrm{U}_{k}$ be the upper Cholesky factors of the process and measurement noise covariances $\mathrm{Q}_{k}$ and $\mathrm{R}_{k}$. The state and Cholesky factors are augmented as

$$
\boldsymbol{x}_{k \mid k}^{a}=\left[\begin{array}{c}
\boldsymbol{x}_{k \mid k} \\
\mathbf{0}
\end{array}\right] \quad \mathrm{S}_{k \mid k}^{a}=\left[\begin{array}{cc}
\mathrm{S}_{k \mid k} & 0 \\
0 & \mathrm{~T}_{k}
\end{array}\right]
$$

\footnotetext{
${ }^{1}$ The general SRUKF does though achieve $O\left(N^{2}\right)$ complexity for parameter estimation [6].
}

and these are used to select a set of sigma points which represent the probability distribution:

$$
\left[\begin{array}{c}
\chi_{0} \\
: \\
\chi_{i} \\
: \\
\chi_{N_{a}+i} \\
:
\end{array}\right]=\left[\begin{array}{c}
\boldsymbol{x}_{k \mid k}^{a} \\
: \\
\boldsymbol{x}_{k \mid k}^{a}+\gamma_{a}\left[\mathrm{~S}_{i}^{a}\right]^{\top} \\
: \\
\boldsymbol{x}_{k \mid k}^{a}-\gamma_{a}\left[\mathrm{~S}_{i}^{a}\right]^{\top} \\
:
\end{array}\right] i=1, \ldots, N_{a}
$$

where $\gamma_{a}$ is a scale, set to $\sqrt{N_{a} /\left(1-W_{0}\right)} . \mathrm{S}_{i}^{a}$ is the $i^{\text {th }}$ row of $\mathrm{S}_{k \mid k}^{a}$.

Each sigma point is passed through the model of state evolution

$$
\chi_{j}^{-}=\boldsymbol{F}\left(\chi_{j}, \boldsymbol{u}_{k}\right) \quad j=0, \ldots, 2 N_{a}
$$

where the state and noise parameters in (1) have been concatenated into $\chi_{j}$. Note that each $\chi_{j}^{-}$has the dimension $N$ of the original state, not the augmented state. The predicted mean is calculated as

$$
\boldsymbol{x}_{k+1 \mid k}=\sum_{j=0}^{2 N_{a}} W_{j} \chi_{j}^{-}
$$

where the $W_{j}$ are weights, here defined to minimize the fourth order errors for a Gaussian [5]:

$$
W_{0}=1-\frac{N_{a}}{3} \quad ; \quad W_{j}=\frac{1-W_{0}}{2 N_{a}} \quad 1 \leq j \leq 2 N_{a} .
$$

Finding the predicted Cholesky factor involves deriving a set of weighted deviations

$$
\begin{aligned}
& \boldsymbol{e}_{0}=\sqrt{\left|W_{0}\right|}\left(\chi_{0}^{-}-\boldsymbol{x}_{k+1 \mid k}\right) \\
& \boldsymbol{e}_{i}=\sqrt{W_{i}}\left(\boldsymbol{\chi}_{i}^{-}-\boldsymbol{x}_{k+1 \mid k}\right) \quad i=1, \ldots, 2 N_{a}
\end{aligned}
$$

and performing the $O\left(N^{3}\right)$ QR decomposition on the $\left(2 N_{a} \times\right.$ N) matrix B

$$
\mathcal{Q R} \leftarrow \mathrm{B}=\left[\begin{array}{llll}
\boldsymbol{e}_{1} & \boldsymbol{e}_{2} & \ldots & \boldsymbol{e}_{2 N_{a}}
\end{array}\right]^{\top}
$$

The resulting $\left(2 N_{a} \times N\right)$ matrix $\mathcal{R}$ is of the form

$$
\mathcal{R}=\left[\begin{array}{c}
\mathrm{S}^{-} \\
0
\end{array}\right]
$$

where $\mathrm{S}^{-}$has size $(N \times N)$. The predicted factor $\mathrm{S}_{k+1 \mid k}$ is then found using Cholesky updating (or downdating if $\left.W_{0}<0\right)$,

$$
\mathrm{S}_{k+1 \mid k}{ }^{\top} \mathrm{S}_{k+1 \mid k}=\mathrm{S}^{-\top} \mathrm{S}^{-}+\operatorname{sign}\left(W_{0}\right) \boldsymbol{e}_{0} \boldsymbol{e}_{0}^{\top} \text {. }
$$

This process, note, is $O\left(N^{2}\right)$. The special form of $\boldsymbol{e}_{0}$, its exclusion from the $\mathrm{QR}$ decomposition, and the subsequent correction by updating are necessary because, uniquely amongst the weights, $W_{0}$ may be negative. 


\section{B. Predicting measurements}

To ease further explanation it is convenient to regard $\mathrm{S}^{-}$ as the output of a function QRFunc() that takes the $e_{1}, e_{2}$, $\ldots$, as its parameters; and also to regard $\mathrm{S}_{k+1 \mid k}$ as the output of a function ChUpdate $\left(\mathrm{S}^{-}, \operatorname{sign}\left(W_{0}\right), \boldsymbol{e}_{0}\right)$.

The treatment of the measurements follows by analogy using as augmented state and factor

$$
\boldsymbol{x}_{k+1 \mid k}^{b}=\left[\begin{array}{c}
\boldsymbol{x}_{k+1 \mid k} \\
\mathbf{0}
\end{array}\right] \quad \mathrm{S}_{k+1 \mid k}^{b}=\left[\begin{array}{cc}
\mathrm{S}_{k+1 \mid k} & 0 \\
0 & \mathrm{U}_{k}
\end{array}\right] .
$$

The $2 N_{b}+1$ sigma points are found using (4) and, as earlier, predicted measurement are generated $\boldsymbol{\zeta}_{j}=\boldsymbol{H}\left(\boldsymbol{\chi}_{j}^{+}\right)$and their predicted mean $y_{k+1 \mid k}$ found as in (6). The weighted deviations

$$
\begin{aligned}
\boldsymbol{e}_{0} & =\sqrt{\left|W_{0}\right|}\left(\boldsymbol{\zeta}_{0}-\boldsymbol{y}_{k+1 \mid k}\right) \\
\boldsymbol{e}_{i} & =\sqrt{W_{i}}\left(\boldsymbol{\zeta}_{i}-\boldsymbol{y}_{k+1 \mid k}\right) \quad i=1, \ldots, 2 N_{b}
\end{aligned}
$$

are used to derive

$$
\begin{aligned}
\mathrm{S}_{y}^{-} & =\operatorname{QRFunc}\left(\boldsymbol{e}_{1}, \boldsymbol{e}_{2}, \ldots, \boldsymbol{e}_{2 N_{b}}\right) \\
\mathrm{S}_{y} & =\operatorname{ChUpdate}\left(\mathrm{S}_{y}^{-}, \operatorname{sign}\left(W_{0}\right), \boldsymbol{e}_{0}\right) .
\end{aligned}
$$

The cross covariance is found as

$$
\mathrm{P}_{x y}=\sum_{j=0}^{2 N_{b}} W_{j}\left[\boldsymbol{\chi}_{j}^{+}-\boldsymbol{x}_{k+1 \mid k}\right]\left[\boldsymbol{\zeta}_{j}-\boldsymbol{y}_{k+1 \mid k}\right]^{\top}
$$

where again the augmented noise terms are pruned from $\chi_{j}^{+}$. The final steps are

$$
\begin{aligned}
\mathrm{K} & =\mathrm{P}_{x y}\left[\mathrm{~S}_{y}^{\top} \mathrm{S}_{y}\right]^{-1}=\mathrm{P}_{x y} \mathrm{~S}_{y}{ }^{-1} \mathrm{~S}_{y}{ }^{\top} \\
\boldsymbol{x}_{k+1 \mid k+1} & =\boldsymbol{x}_{k+1 \mid k}+\mathrm{K}\left(\boldsymbol{y}_{k+1}-\boldsymbol{y}_{k+1 \mid k}\right) \\
\mathrm{S}_{k+1 \mid k+1} & =\text { SeqChUpdate }\left(\mathrm{S}_{k \mid k+1},-1, \mathrm{KS}_{y}^{\top}\right) .
\end{aligned}
$$

Equation (21) denotes repeated Cholesky updating using successive columns of $\mathrm{KS}_{y}^{\top}$ as the updating vector.

\section{The Square Root UKF APPlied to MonoSLAM}

The application of the Square Root UKF to monoSLAM has thrown out challenges where particular care has been required during implementation to obtain an efficient and effective algorithm. The first is the crucial observation that a significant computational saving can be made at (10), the QR decomposition for the state prediction, which reduces the complexity of the SRUKF from $O\left(N^{3}\right)$ to $O\left(N^{2}\right)$. This saving is possible because the map points do not move in the motion model. The second concerns the initialization of features using the inverse depth method in the SRUKF.

\section{A. Exploiting the form of the state evolution}

Because the map points do not move, the QR decomposition of (10) can be abbreviated. When written out in component form the sigma points for state prediction are

$$
\begin{aligned}
\chi_{0, j} & =\left(x_{k \mid k}^{a}\right)_{j} \\
\chi_{i, j} & =\left(x_{k \mid k}^{a}\right)_{j}+\gamma_{a} \mathrm{~S}_{i, j}^{a} \quad 1 \leq i, j \leq N_{a} \\
\chi_{N_{a}+i, j} & =\left(x_{k \mid k}^{a}\right)_{j}-\gamma_{a} \mathrm{~S}_{i, j}^{a}
\end{aligned}
$$

where $\mathbf{S}_{i, j}^{a}=0$ if $i>j$. We order the state vector with the map points first ( $3 m$ entries), followed by the camera ( $c$ entries) and lastly the the augmented noise terms ( $d$ entries), so that $N=3 m+c$ and $N_{a}=3 m+c+d$. Then each sigma point is put through the update function $\boldsymbol{F}$.

Again because the map points do not move, the top $3 \mathrm{~m}$ elements remain unchanged. These are, for $1 \leq i \leq N_{a}$ and $1 \leq j \leq 3 m$

$$
\begin{aligned}
\chi_{0, j}^{-} & =\chi_{0, j}=\left(x_{k \mid k}^{a}\right)_{j} \\
\chi_{i, j}^{-} & =\chi_{i, j}=\left(x_{k \mid k}^{a}\right)_{j}+\gamma_{a} \mathrm{~S}_{i, j}^{a} \\
\chi_{N_{a}+i, j}^{-} & =\chi_{N_{a}+i, j}=\left(x_{k \mid k}^{a}\right)_{j}-\gamma_{a} \mathrm{~S}_{i, j}^{a} .
\end{aligned}
$$

Those that change are for $0 \leq i \leq 2 N_{a}$ and $(3 m+1) \leq j \leq$ $N$ :

$$
\chi_{i, j}^{-}=F_{j}\left(\chi_{i}\right) .
$$

Now consider the elements of $\boldsymbol{x}_{k+1 \mid k}^{a}$ :

$$
\left(x_{k+1 \mid k}\right)_{j}=\sum_{i=0}^{2 N_{a}} W_{i} \chi_{i, j}^{-} .
$$

For $1 \leq j \leq 3 m$, the summation over index $i$ causes the $\pm \gamma_{a} \mathrm{~S}_{i, j}^{a}$ terms to cancel, so that

$$
\left(x_{k+1 \mid k}\right)_{j}=\left(x_{k \mid k}\right)_{j} \quad 1 \leq j \leq 3 m
$$

but

$$
\left(x_{k+1 \mid k}\right)_{j} \neq\left(x_{k \mid k}\right)_{j} \quad(3 m+1) \leq j \leq N .
$$

The elements $1 \leq j \leq 3 m$ of vectors $\boldsymbol{e}_{i}\left(1 \leq i \leq N_{a}\right)$ are:

$$
\begin{aligned}
\left(e_{0}\right)_{j} & =\sqrt{\left|W_{0}\right|}\left(\chi_{0, j}^{-}-\left(x_{k+1 \mid k}\right)_{j}\right)=0 \\
\left(e_{i}\right)_{j} & =\sqrt{W_{i}}\left(\chi_{i, j}^{-}-\left(x_{k+1 \mid k}\right)_{j}\right) \\
& =+\sqrt{W_{i}} \gamma_{a} \mathrm{~S}_{i, j}^{a} \\
\left(e_{N_{a}+i}\right)_{j} & =\sqrt{W_{i}}\left(\chi_{N_{a}+i, j}^{-}-\left(x_{k+1 \mid k}\right)_{j}\right) \\
& =-\sqrt{W_{i}} \gamma_{a} \mathrm{~S}_{i, j}^{a} .
\end{aligned}
$$

But as noted above $\mathrm{S}^{a}$ is upper triangular, so that for $1 \leq$ $j \leq 3 m$

$$
\begin{array}{r}
\mathrm{B}_{i, j}=\left(e_{i}\right)_{j}= \begin{cases}+\sqrt{W_{i}} \gamma_{a} \mathrm{~S}_{i, j}^{a} & 1 \leq i \leq j \\
0 & (j+1) \leq i \leq N_{a}\end{cases} \\
\mathrm{B}_{N_{a}+i, j}=\left(e_{N_{a}+i}\right)_{j}= \begin{cases}-\sqrt{W_{i}} \gamma_{a} \mathrm{~S}_{i, j}^{a} & 1 \leq i \leq j \\
0 & (j+1) \leq i \leq N_{a}\end{cases}
\end{array}
$$

The form of this sparse $B$ is shown in Figure 1(a). To recover $\mathrm{S}^{-}$we could perform a $\mathrm{QR}$ decomposition on $\mathrm{B}$ and use $\mathcal{R}$. However the form of B, shown in Figure 1(a), can be further simplified to that of Figure 1(b) by pre-multiplying with the orthonormal matrix

$$
Q_{1}=\left[\begin{array}{cccc}
-I_{1} / \sqrt{2} & 0 & I_{1} / \sqrt{2} & 0 \\
0^{\top} & I_{2} & 0^{\top} & 0 \\
I_{1} / \sqrt{2} & 0 & I_{1} / \sqrt{2} & 0 \\
0^{\top} & 0 & 0^{\top} & I_{2}
\end{array}\right]
$$

where $\mathrm{I}_{1}$ is a $3 \mathrm{~m}^{2}$ identity, $\mathrm{I}_{2}$ is a $(c+d)^{2}$ identity, and 0 is a $3 m \times(c+d)$ or a $(c+d)^{2}$ zero matrix. The $\mathcal{R}$ from the 
$e_{0}$ only has non zero elements in the fourth, fifth and sixth elements from the bottom. This means that the downdating algorithm can simply be stopped before the last three rows of the factor. This is because they will always be entirely filled with zeros as in (40).

Once the depth estimate of a feature has converged it is converted to a $3 \mathrm{D}$ point feature. This is a straightforward application of the square root unscented transform to the state and covariance. In the QR decomposition the sigma point matrix can be pre-multiplied by an orthonormal matrix as was done in (36).

There are also special issues in deleting features from the SRUKF which appear neither in the EKF nor UKF. A description is given elsewhere [16].

\section{EXPERIMENTAL COMPARISONS}

The experiments carried out demonstrate three things: first that the results from the UKF and the SRUKF are all but identical; that the SRUKF provides greater consistency than the EKF, but that both still suffer from inconsistency; and lastly that using the square root form reduces the complexity of the UKF to $O\left(N^{2}\right)$, but still at greater cost than the EKF.

Each algorithm has been implemented in $\mathrm{C}++$ on a 2.66 GHz Intel Core 2 Duo Processor running Fedora Core 5. Care has been taken to produce efficient code using proven optimized libraries, but no extraordinary effort has been used to produce highly optimized code in one filter at the expense of another.

The filters run either from real imagery provided by a Firewire camera, or from synthetic data read in from filestore. In this paper we report only on the use of synthesized data, as it provides reproducible ground truth for comparison of compute time, complexity, accuracy, and consistency.

\section{A. Filter comparability}

A useful measure of filter performance is consistency. A filter is consistent if the state estimate $\boldsymbol{x}_{k \mid k}$ is unbiased, so that $E\left[\hat{\boldsymbol{x}}_{k \mid k}-\boldsymbol{x}_{k}\right]=\mathbf{0}$ where $\boldsymbol{x}_{k}$ is ground truth, and if the normalized estimation error squared (NEES), or squared Mahalanobis distance, is bounded appropriately for a $\chi^{2}$ distributed variable with $N$ degrees of freedom ( $N$ is the state's dimension) at some chosen confidence level

$$
\epsilon_{k}^{2}=\left[\boldsymbol{x}_{k \mid k}-\boldsymbol{x}_{k}\right]^{\top} \mathrm{P}_{k}^{-1}\left[\boldsymbol{x}_{k \mid k}-\boldsymbol{x}_{k}\right] \in\left[b_{\mathrm{L}}, b_{\mathrm{U}}\right] .
$$

We compare the EKF, UKF and SRUKF by recording the NEES for the camera pose when it moves through various scenes of distributed 3D points. The synthetic camera is a model of that used for live experiments, with a field of view of $80^{\circ} \times 55^{\circ}$ and a notional $640 \times 480$ pixel image, though it omits radial distortion. To generate measurements, 3D points were projected into the image, and their coordinates corrupted by Gauss random measurement noise with a standard deviation of 0.5 pixel. For each experiment the results of 50 runs each 500 frames long were averaged.

Fig. 2 shows a plot of the normalized difference between the NEES scores of the UKF and SRUKF during a typical run. The difference is of order $10^{-6}$, and there are no systematics to suggest subtle performance differences. Further

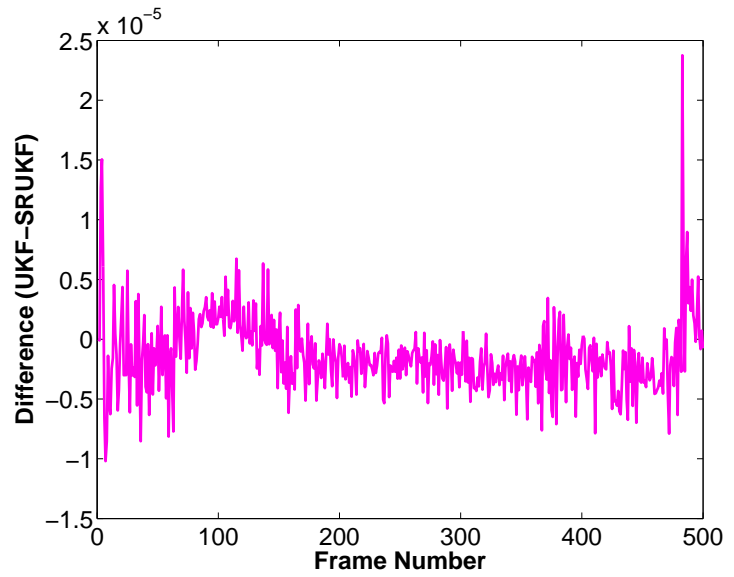

Fig. 2. A plot of the typical the normalized difference in the NEES scores between the UKF and the SRUKF. In this run as in others, the difference is typically of order $10^{-6}$.

comparison between the filters now need involve only the SRUKF and EKF.

Fig. 3 shows two comparisons of the NEES for these filters. In the first, the camera moves past an array of scene of points in a planar grid with constant translational velocity and zero angular velocity, a motion which conforms to the model. The plots of Figure 3(a) show clearly that the SRUKF outperforms the EKF, but nonetheless both filters become inconsistent after several tens of frames. The degree of overconfidence (i.e. the degree to which $\mathrm{P}$ is underestimated) in both filters grows steadily over time.

In the second example, the camera tumbles through a constellation of points scattered randomly within a cube region. At each timestep the camera undergoes a random acceleration of $0.5 \mathrm{~m} \mathrm{~s}^{-2}$ and $0.5 \mathrm{rad} \mathrm{s}^{-2}$, half of that assumed by the motion model. Figure 3(b) shows the resulting NEES values. Again the SRUKF out-performs the EKF, though again both filters become inconsistent.

\section{B. Algorithmic complexity}

The second set of trials explores the comparative speed of three algorithms, and determines their complexity as a function of increasing state size. To eliminate irrelevant overheads such as disk access, computing times were derived separately for the stages of (i) calculating the prior, (ii) projecting the search ellipses into the synthetic image, which involves calculating the innovation covariance, and (iii) calculating the posterior, and then summed. The resulting computing times are shown as functions of state size in Fig. 4.

Below a state size of around 100, all three methods are dominated by a mix of fixed time and $O(N)$ operations, with a resulting complexity of about $O\left(N^{0.5}\right)$. Beyond this size, equivalent to greater than thirty or so points in the map, both the EKF and SRUKF tend to $O\left(N^{2}\right)$ complexity. The EKF, however, is evidently a factor of around 10 to 16 times faster. Above a state size of some 200 the UKF becomes significantly more costly than the SRUKF, and tends to $O\left(N^{3}\right)$ complexity. In our experiments at a state size of 2300 (770 3D points), the UKF has reached $O\left(N^{2.8}\right)$. 


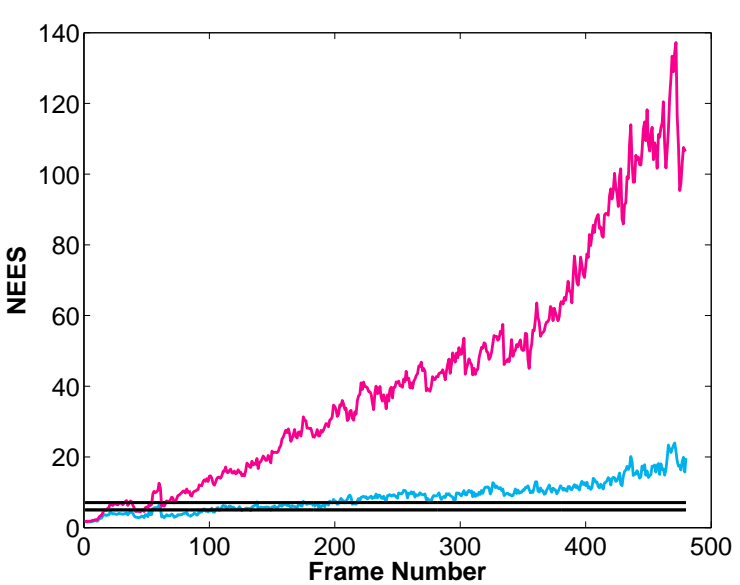

(a)

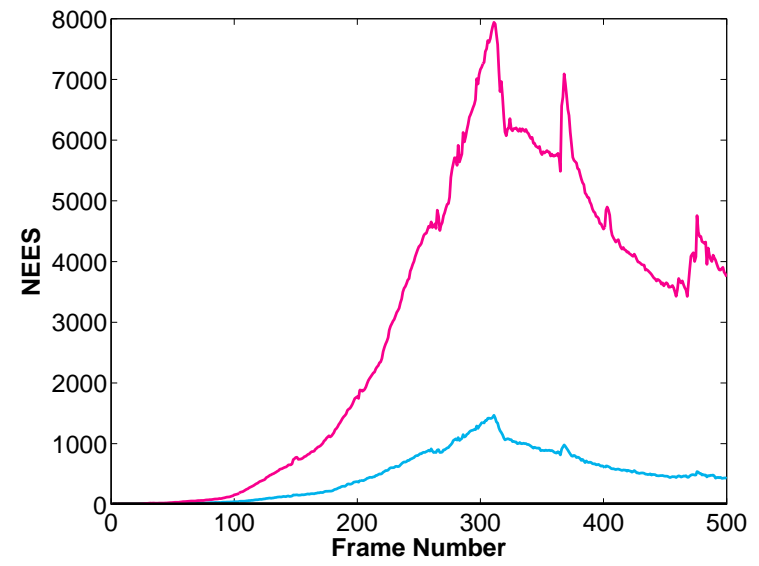

(b)

Fig. 3. The NEES score for the EKF (upper curve) and the SRUKF (lower) for (a) a constant velocity linear motion past a grid of points and (b) a tumbling motion through randomly sited 3D points. In (a) are shown are the upper and lower bounds of the $\chi^{2}$ consistency test. At the scale of (b) these are indistinguishable from the $x$-axis.

\section{CONCLUSIONS AND FUtURE WORK}

This paper has introduced a Square Root Unscented Kalman Filter for visual monocular SLAM. It has been shown how the general Square Root formulation can be modified for the SLAM problem to obtain an $O\left(N^{2}\right)$ implementation, rather than the usual $O\left(N^{3}\right)$ complexity of the general SRUKF and UKF. It has also been shown how to initialize inverse depth features in the SRUKF. The results from experiments on synthetic data with known ground truth demonstrate that, as expected, the UKF and SRUKF perform identically in terms of the quality of the structure and motion output. Comparisons of consistency show that the SRUKF (and hence UKF) outperform the EKF under a variety of motions, but also show that both types of filter repeatedly underestimate the size of the covariance matrix. It has been demonstrated experimentally that the complexity of our SRUKF-based SLAM is indeed $O\left(N^{2}\right)$ and the same as the EKF, but that the execution time of the SRUKF remains stubbornly an order of magnitude higher than that of the EKF. Although we have a video rate implementation of the SRUKF, work is ongoing to see if the order of magnitude performance hit over the EKF can be reduced.

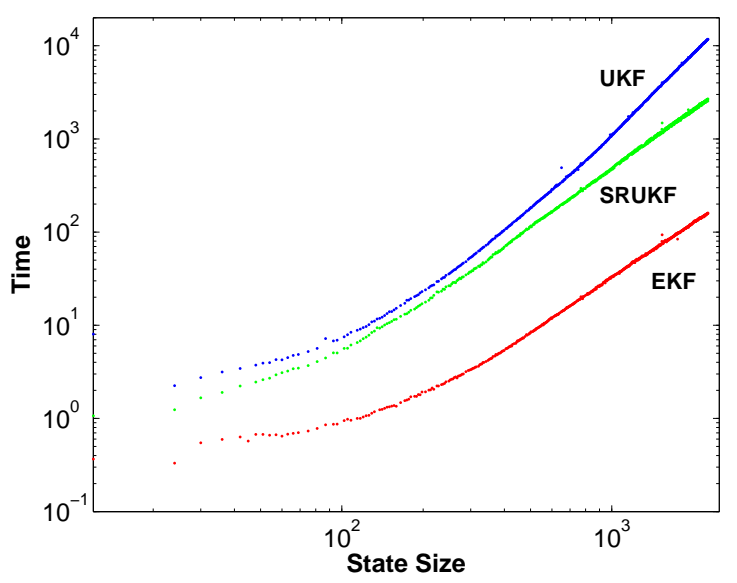

Fig. 4. Log-log plots of the total times (in seconds) taken for the core "Kalman" stages of the UKF, SRUKF and EKF versus the size of the state. The occasional timing blibs are due to systems programs which could not be switched off.

\section{REFERENCES}

[1] N. Ayache and O.D. Faugeras. Maintaining representations of the environment of a mobile robot. IEEE Trans. Robotics and Automation, 5(6):804-819, 1989.

[2] C.G. Harris and J.M. Pike. 3d positional integration from image sequences. Image and Vision Computing, 6(2):87-90, 1987.

[3] R.C. Smith and P. Cheeseman. On the representation and estimation of spatial uncertainty. Int. Journal of Robotics Research, 5(4):56-68, 1986.

[4] J.J. Leonard and H.F. Durrant-Whyte. Directed Sonar Sensing for Mobile Robot Navigation. Kluwer Academic, Boston MA, 1992.

[5] S. J. Julier and J. K. Uhlmann. Unscented filtering and nonlinear estimation. Proceedings of the IEEE, 92(3), 2004.

[6] R. Van der Merwe and E Wan. The square-root Unscented Kalman filter for state and parameter estimation. In IEEE International Conference on Acoustics, Speech, and Signal Processing, Salt Lake City, Utah, 2001, volume 6, pages 3461 - 3464, 2001.

[7] T. Lefebvre, H. Bruyninckx, and J de Schutter. Kalman filters for nonlinear systems: A comparison of performance. International Journal of Control, 77(7):639-653, 2004.

[8] S. J. Julier, J. K. Uhlmann, and H.F Durrant-Whyte. A new approach for filtering nonlinear systems. In The Proceedings of the American Control Conference, Seattle, Washington, 1995, pages 1628-1632, 1995.

[9] R.Martinez-Cantin and J. A. Castellanos. Unscented SLAM for largescale outdoor environments. In Proc IEEE/RSJ Conf on Intelligent Robots and Systems, Edmonton, Canada, August 2-6, 2005, pages 3427-3432, 2005.

[10] J. Andrade-Cetto, T. Vidal-Calleja, and A. Sanfeliu. Unscented transformation of vehicle states in SLAM. In Proc Int Conf on Robotics and Automation, Barcelona, Spain, Apr 18-22, 2005, pages 323-328, 2005.

[11] J. Langelaan and S. Rock. Passive GPS-Free navigation for small UAVs. In IEEE Aerospace Conference, Big Sky MT USA 2005, pages 1-9, 2005.

[12] D. Chekhlov, M. Pupilli, W. Mayol-Cuevas, and A. Calway. Realtime and robust monocular SLAM using predictive multi-resolution descriptors. In Proc 2nd Int Symp on Visual Computing, Lake Tahoe Nevada, November 6-8, 2006, pages 276-285, 2006.

[13] A.J. Davison, I.D. Reid, N. Molton, and O. Stasse. MonoSLAM: Realtime single camera SLAM. IEEE Transactions on Pattern Analysis and Machine Intelligence, 29(6):1052-1067, 2007.

[14] J.M.M. Montiel, J. Civera, and A.J. Davison. Unified inverse depth parametrization for monocular SLAM. In Robotics: Science and Systems, Philadelphia, Pennsylvania, August 16-19, 2006., 2006.

[15] N. J. Higham. Analysis of the Cholesky decomposition of a semidefinite matrix. In M. G. Cox and S. J. Hammarling, editors, Reliable Numerical Computation, pages 161-185. Oxford University Press, 1990.

[16] Steven Holmes. Feature management for monocular visual SLAM. PRS Transfer Report, Department of Engineering Science, University of Oxford, September 2007. 